\title{
Le traitement macrostructural de l'emprunt au français dans les dictionnaires locaux au Gabon
}

\author{
Edgard Maillard Ella, Institut de Recherche en Sciences Humaines, \\ Centre National de la Recherche Scientifique et Technologique, \\ Libreville, Gabon (ellamaillard@yahoo.fr)
}

\begin{abstract}
Résumé: Les populations locales empruntent souvent de façon pragmatique aux autres langues les mots dont elles ont besoin dans les leurs. Cela est plus applicable lorsque deux populations de langues différentes se côtoient comme c'est le cas avec français et les langues locales du Gabon. L'emprunt est un processus inconscient et un facteur constitutif de la vie de toutes les langues du monde. C'est pour cela que bien que moins nombreux que les mots locaux, les mots d'emprunt sont très courants dans leur vocabulaire. Certains de ces emprunts sont remplacés par des termes locaux lorsque les populations locales se sont familiarisées avec les concepts ou les choses véhiculés par les mots étrangers. Les uns sont gardés tels quels, et d'autres sont morpho-phonologiquement modifiés afin d'être adaptés aux caractéristiques linguistiques locales. Un dictionnaire est confectionné en fonction des usagers. C'est ainsi que le lexicographe adopte à son tour cette approche pragmatique pour que le public se reconnaisse dans l'ouvrage produit. Pendant le traitement macrostructural quand les mots à inclure comme lemmes sont choisis, il incorpore dans un premier temps les emprunts utilisés par les populations locales. Progressivement, il incorporera dans les éditions revues et corrigées, les termes locaux qui ont remplacé certains emprunts ainsi que les modifications morpho-phonologiques subies par les autres. Dans ce travail, nous voudrions suggérer cette approche pragmatique aux personnes qui confectionnent les dictionnaires au Gabon et qui sont souvent confrontées à l'absence de plusieurs mots modernes dans les langues locales. Ce travail va à l'encontre de la création lexicale qui est connue comme étant une approche puriste et qui s'avère souvent être laborieuse et incertaine. Nous voudrions montrer que l'approche pragmatique qui incorpore les deux approches, constitue une méthodologie qui présente plus d'avantages que une approche puriste. Le dialecte fang ntoumou de Bitam est utilisé ici comme exemple pour étayer ce travail qui est applicable à toutes les autres langues locales du Gabon.
\end{abstract}

Mots clés: APPROCHE PRAGMATIQUE, APPROCHE PURISTE, CREATION LEXICALE, DICTIONNAIRE, EMPRUNT, FRANÇAIS, LANGUES LOCALES

\begin{abstract}
The Macrostructural Treatment of Borrowings from French in Local Gabonese Dictionaries. Local populations often pragmatically borrow words from other languages, which they need in theirs. This applies mostly when two populations with different languages come in contact as is the case with French and the local languages of Gabon. Borrowing is an unconscious process and an essential factor in the life of all the world's languages. This is why, although they are less numerous than local words, borrowed words are very common in their vocabularies. Some of these borrowings are replaced by local terms when local populations become
\end{abstract}

Lexikos 23 (AFRILEX-reeks/series 23: 2013): 88-112 
familiar with the concepts or things referred to by the foreign words. Some are retained as they are and others are morphophonologically modified in order to adapt to local linguistic characteristics. A dictionary is compiled in accordance with the users. This is why the lexicographer in his turn adopts this pragmatic approach so that the users will identify themselves with the finished work. During the macrostructural treatment when words to include as lemmas are chosen, he initially incorporates borrowings used by the local population. Gradually in revised and corrected editions, he will incorporate local terms which have replaced certain borrowings as well as morphophonological changes undergone by others. In this article, we want to suggest this pragmatic approach to persons who compile dictionaries in Gabon and who are often faced with the absence of many modern words in the local languages. This article goes against lexical creation which is known as being a purist approach and which often proves to be laboured and dubious. We want to show that a pragmatic approach, which incorporates both approaches establishes a methodology, which presents more advantages than a purist approach. The dialect Fang Ntumu of Bitam is used here as an example to support this article, which is suitable for all the other local languages of Gabon.

Keywords: BORROWING, DICTIONARY, FRENCH, LEXICAL CREATION, LOCAL LANGUAGES, PRAGMATIC APPROACH, PURISTIC APPROACH

\section{Introduction}

L'une des nécessités de confectionner des dictionnaires au Gabon aujourd'hui répond au besoin de standardiser les différents lexiques des langues locales. Il y a en effet une volonté de l'État de faire en sorte qu'à l'instar du français, ces langues puissent être également enseignées à l'école ${ }^{1}$. Or, dès qu'une langue doit être enseignée, son lexique doit nécessairement s'élargir et se moderniser.

Plusieurs personnes s'engagent actuellement à confectionner des dictionnaires dans ce sens. Il importe donc d'attirer l'attention des uns et des autres sur les risques d'une pratique lexicographique désordonnée. La confection des dictionnaires au Gabon doit se faire selon les règles de l'art afin de produire des ouvrages de bonne qualité. Comme le souligne Gouws (2001: 96), la théorie de la lexicographie n'est pas simplement une théorie pour une théorie, c'est une théorie qui permet d'améliorer la qualité des dictionnaires.

Par ailleurs, il est également nécessaire de souligner l'importance, le rôle et la contribution des locuteurs dans cette entreprise. En effet, d'aucuns semblent souvent oublier que c'est une image d'Epinal, comme nous le faisons souvent assez clairement observer dans nos travaux, que la langue appartient aux locuteurs. Ces derniers ne servent pas que de simples informateurs, mais ce sont bel et bien les principaux acteurs de la langue car ce sont eux qui la façonnent depuis la nuit des temps. La minorité compétente de grammairiens, linguistiques et lexicographes qui pensent qu'ils auraient le pouvoir de statuer sur la langue ne font en réalité que décrire, organiser et standardiser la façon dont les locuteurs pratiquent leurs langues. À ce titre, il est obligatoire de les prendre en compte dans chaque aspect du traitement des langues ainsi que de leur développement. Diop (1979: 345) souligne justement à ce propos que le développement 
d'une langue est une entreprise de longue haleine qui est tantôt spontanée, tantôt confiée à des experts, sans que les deux modalités s'excluent.

Le lexicographe ${ }^{2}$ est conscient de ce fait car le dictionnaire est un ouvrage de base qui a une validité sociale acquise et une reconnaissance sociale consacrée. C'est pour cela que celui-ci va vers ses publics, il en est, cf. Kalonji Zezeze (1993: 42). Il publie donc le dictionnaire pour que ce soit un ouvrage dans lequel le public puisse se reconnaître.

C'est à ce propos que dans ce travail, nous nous appuyons sur la façon pragmatique avec laquelle les populations locales empruntent aux autres langues les mots qui manquent dans les leurs. Cette pratique linguistique des populations, destinée à développer et moderniser leurs langues, est le fondement de l'approche pragmatique que nous suggérons dans ce travail. Il s'agit $\mathrm{du}$ traitement macrostructural des emprunts au français dans les dictionnaires locaux au Gabon. L'approche pragmatique que nous proposons dans ce travail n'est pas une approche nouvelle. Madiba (2001) l'a suggérée comme modèle pour la modernisation de la terminologie des langues sud africaines. Joseph (1984, cité dans Madiba 2001: 64) l'a recommandée dans la standardisation d'une langue. Jernudd (1977, cité dans Madiba 2001: 64) qui critique l'approche puriste du développement terminologique de l'Hébreu, fait également appel à certaines réalités linguistiques qui sont pragmatiques. Marshad (1984) et Mwansoko (1990) ont, quant à eux, proposé l'approche pragmatique dans la modernisation du Swahili.

Pour faire ressortir l'intérêt, les objectifs et les enjeux de ce travail, nous allons d'abord commencer par présenter l'approche puriste qui prône la création lexicale dans le développement et la modernisation de la terminologie des langues. Nous allons nous appuyer sur les travaux de Madiba (2001) et nous allons naturellement contextualiser l'approche puriste dans le cas précis du Gabon. C'est dans cette même partie et en nous référant toujours à Madiba (2001) que nous examinerons également les limites de cette approche.

Dans une seconde partie et en nous renvoyant encore une fois encore au travail de Madiba (2001), nous discuterons de l'approche pragmatique qui est en faveur de l'emprunt direct à une autre langue pour le développement et la modernisation de la terminologie d'une langue. En prenant des exemples concrets de l'emprunt au français dans le dialecte fang ntoumou de Bitam, nous allons montrer qu'emprunter au français dans le traitement de la macrostructure des dictionnaires locaux est conforme à la pratique linguistique des populations locales. En sus de présenter plus d'avantages que l'approche puriste, nous ferons aussi observer que l'approche pragmatique de la collecte des données destinées à la macrostructure incorpore l'approche puriste qui, au fil des années, se déroule de façon plus naturelle. Nous verrons par ailleurs que cette approche est l'application d'un aspect du processus lexicographique. L'approche pragmatique aboutira à la production de dictionnaires locaux au Gabon caractérisés par des dispositifs spécifiques correspondant aux principes de la métalexicographie tels qu'énoncés par Wiegand (Smit 1996). Il s'agit de la prévisibilité, de la calculabilité, de l'analysabilité et de la contrôlabilité. 


\section{L'approche puriste dans le traitement de la macrostructure}

\subsection{Approche définitoire et les raisons}

Nous définirons l'approche puriste, dans le traitement de la macrostructure des dictionnaires africains en général et gabonais en particulier, comme le recours immédiat à la création lexicale en vue d'élaborer les mots n'existant pas dans les langues locales. Cette approche exclut tout emprunt à la langue officielle européenne en particulier. C'est le cas du français au Gabon.

De nombreuses personnes qui confectionnent les dictionnaires dans ce pays sont souvent fortement motivées par le désir de conserver leurs langues et cultures locales en éradiquant et en évitant d'utiliser les mots du français en particulier. Ces mots sont considérés comme des impuretés dont l'usage aura pour conséquence la perte et l'aliénation de l'identité des langues et cultures locales. Cette approche est donc souvent légitimée par des critères idéologiques, politiques, sociaux et émotifs. Elle n'est pas un fait nouveau car elle a été en vigueur France par exemple qui a longtemps considéré l'influence de l'anglais sous cet angle, cf. Weinstein (1989). C'est ainsi que dans le processus de confection de leurs dictionnaires, plusieurs lexicographes gabonais excluent automatiquement dans le choix des items lexicaux à inclure, comme lemmes ou entrées de nos dictionnaires, de nombreux mots français pourtant déjà couramment utilisés, connus et familiers aux populations.

Or, faute des équivalents en langues locales pour le moment, les mots empruntés au français sont naturellement utilisés dans les conversations des populations. C'est ainsi qu'en fang ntoumou de Bitam, il n'est pas rare d'entendre par exemple3:

- Ma ke a banque a ke a gnong crédit akala a kousse metoua ya a long nda.

(Je vais à la banque prendre un crédit pour acheter une voiture et construire une maison.)

- Ma ke a kissouane a ke a kousse be carreaux ya ciment akala e nda ma long. (Je vais en ville pour aller acheter des carreaux et du ciment pour la maison que je construis.)

- Be ve nommer e nome-ngouane wôme Ministre e Conseil be Ministres a ve lote ongwuéghé.

(On a nommé mon beau-fils Ministre lors du Conseil de Ministres qui s'est tenu hier.)

Pourtant aucune impureté ne transparaît lorsque les mots français sont si normalement utilisés dans les conversations quotidiennes dans les langues locales du Gabon comme nous le voyons dans cet exemple avec le fang ntoumou de Bitam. Il en sera de même à l'écrit, comme nous venons de le transcrire. Considérer les mots d'une langue étrangère mais connue et familière comme des éléments impurs et des facteurs de dysfonctionnement et d'aliénation de l'iden- 
tité des langues et cultures locales du Gabon n'est pas une approche objective. Tout au contraire, celle-ci est contre nature car l'emprunt est un processus inconscient et un facteur constitutif de la vie de toutes les langues du monde. En effet, nous pouvons considérer que ce processus linguistique résulte de la capacité de l'instinct de survie de l'homme à s'adapter à son environnement politique, économique et social sans cesse en mutation en utilisant autant qu'il peut des éléments qui sont à disposition. Dans le cas de la langue, les emprunts font partie de ces éléments.

Pour revenir au cas de la France, nous pensons que l'invasion des mots de l'anglo-américain particulièrement, n'est qu'un refoulement qui a été et est plutôt révélateur d'un conflit d'intérêt entre superpuissances en lutte pour la suprématie mondiale. Dans ce contexte, l'influence de la langue issue d'un autre pays est peut-être considérée comme un signe de faiblesse ou d'infériorité. Cette approche puriste est vaine car le développement de la technologie et la domination de l'anglo-américain dans les sciences et les techniques actuelles confère une suprématie considérable de la langue anglaise à l'échelle planétaire qui n'est pas subie que par la seule langue française. L'anglais est devenu la lingua franca du monde contemporain, c'est-à-dire la langue véhiculaire des communications internationales, tant sur le plan commercial que culturel, scientifique, technologique, diplomatique et politique. Pour preuve, de nombreux programmes télévisés en France et à forte audience présentent des titres en anglais en vue d'être plus accrocheurs: Money drop et Secret Story qui sont des programmes télévisés d'une des chaînes publiques françaises, à savoir TF1.

$\mathrm{Au}$ Gabon et dans l'arrière-pays, grande fut notre surprise en lisant «Air Force One» comme enseigne d'un snack bar (encore une fois un anglicisme) à Ovan, dans le Département de la Mvoung, province de l'Ogooué-Ivindo. Il est évident que le «Air Force One» d'Ovan n'est pas le seul établissement commercial au Gabon portant un nom anglais. C'est dire combien de fois la pratique langagière qui est inconsciente, obéit naturellement à des besoins prosaïques tels que le business (encore un anglicisme) comme dans le cas que nous venons d'illustrer. En empruntant naturellement à une autre langue un mot qui lui semble plus approprié pour satisfaire un quelconque besoin, l'homme fait que la langue transgresse les considérations émotives, idéologiques et sociopolitiques qui sont contre nature dans le processus de la pratique linguistique.

Dans le cas du Gabon, à l'instar de la plupart des pays d'Afrique noire francophone, l'approche puriste est justifiée par un élan de patriotisme ou de nationalisme peut-être parfois exacerbé. Celui-ci débouche sur une idéologie anti langue française dans le processus de développement des langues locales. Cette approche résulte aussi de la volonté d'ériger ou de rétablir une forme d'indépendance face à l'invasion de la culture, de la politique, du commerce ainsi que de la technologie de l'ancienne puissance colonisatrice occidentale comme le souligne Madiba (2001: 56). Cependant, comme nous avons tenté de le démontrer, l'approche puriste va à l'encontre d'un processus inconscient et naturel de la langue dans l'aspect du développement et de la modernisation de la terminologie. 


\subsection{Les limites de l'approche puriste}

\subsubsection{La menace de l'intégrité de l'identité culturelle et de la souveraineté nationale}

L'un des arguments ou le seul qui revient régulièrement de la part des personnes qui prônent l'approche puriste dans le traitement de la macrostructure lors de la confection de dictionnaires gabonais, demeure l'aliénation ou purement et simplement la perte de l'intégrité de notre identité culturelle et de notre souveraineté nationale. Nous pouvons dire que cette crainte n'est pas à redouter si nous nous référons à l'histoire des autres langues du monde. C'est le cas de la langue anglaise dont $50 \%$ à $60 \%$ du vocabulaire est d'origine française et latine, cf. SALIC (l'Histoire de la langue française). Cette langue qui est la plus diffusée au monde aujourd'hui n'a pas souffert d'une perte de son identité et celle de la culture de ces locuteurs

C'est aussi le cas de la langue française. L'identité culturelle et de la souveraineté nationale de la France sont intactes quand bien même plusieurs mots proviennent d'autres langues. Henriette Walter et Gérard Walter, deux linguistes lexicologues d'origine française, ont effectué une analyse minutieuse de 70000 termes puisés dans les dictionnaires Le Petit Larousse et Le Petit Robert, cf. SALIC (l'Histoire de la langue française 23). Cette analyse révèle que 8088 mots proviennent des emprunts aux langues étrangères, soit $11 \%$ du corpus. Ce pourcentage est inférieur à la réalité si d'une part, le latin et le grec sont pris en compte. Et d'autre part, si les lexiques spécialisés d'origine anglaise sont considérés dans cette analyse compte tenu du développement de la technologie et la domination de l'anglo-américain dans les sciences et les techniques actuelles. Cette étude montre par la même occasion que le nombre des langues prêteuses s'élève à plus de 120. Il s'agit entre autres du latin, du grec, de l'anglais, de l'italien, de l'espagnol, du hollandais, de l'arabe et même du swahili, du coréen et de l'iranien. C'est dire que si l'emprunt de mots à une ou plusieurs langues étrangères constituait un facteur d'aliénation et d'intégrité de l'identité culturelle et la souveraineté nationale d'un pays, la France et l'Angleterre seraient aujourd'hui des pays ayant une identité culturelle et une souveraineté nationales peut-être inexistantes. Ce qui est évidemment loin d'être le cas.

C'est dire que l'intégrité de l'identité culturelle et de la souveraineté nationale du Gabon ne pèsent sous aucune menace d'être aliénées si les mots sont empruntés au français pour combler le manque de mots modernes qui existe dans les langues locales. Il n'y aura donc aucun mal à ce que, dans le traitement dictionnairique, ces mots soient inclus comme lemmes ou entrées dans la macrostructure des ouvrages locaux. Il n'en sera pas moins une hérésie scientifique dans cette démarche lexicographique ${ }^{4}$.

\subsubsection{Lenteur, difficultés d'adoption et refoulement des termes créés}

La confection de dictionnaires constitue une activité laborieuse et de très 
longue haleine. Il est connu que Pierre Larousse (1817-1875) est mort bien avant d'avoir vu son dictionnaire terminé, tant on se rend rarement compte du fait que les quantités de travail et de temps à consacrer à la production de ce livre sont énormes. Si l'approche puriste qui consiste à créer les mots qui n'existent pas dans les langues locales du Gabon pour la confection des dictionnaires locaux au Gabon est adoptée, cela signifie qu'il faut s'engager à créer quelques dizaines de milliers de termes dans des domaines autant nombreux que diversifiés. Pour montrer l'ampleur de la tâche à accomplir, il s'agit entre autres des termes provenant de l'économie, de la politique, du social, de l'administration, de la justice, des finances, du commerce, de l'industrie, des sciences exactes et humaines, des techniques, de l'informatique, de l'électronique, de la mécanique, de la médecine, de la télévision, du sport, de la drogue, de la violence, de l'armement, de la culture, de l'histoire, de l'environnement, du cinéma, de la musique, de l'architecture, de l'art, de la danse et du tourisme pour ne citer que ceux-là. Il existe bien d'autres domaines encore.

Cette activité constituerait un énorme travail mais qui serait tout à fait réalisable. Mais à quel moment se terminera-t-il et comment sera-t-il possible de gérer le déferlement de mots qui se créent tous les jours à cause d'une société sans cesse en développement et en mutation? C'est pour cette raison que les dictionnaires de langue tels que ceux produits par les éditions Larousse, se voient aujourd'hui obligés de produire une édition chaque année, ce qui n'était pas le cas il y a de cela quelques décennies, non pas pour des raisons commerciales mais pour fournir aux utilisateurs le lexique le plus actualisé possible. À cela, il faut encore ajouter le fait que les populations locales prendront nécessairement du temps pour adopter les termes créés même si ces derniers proviennent de leurs langues-mères. Pire, elles peuvent tout simplement les rejeter comme le montrent les travaux des auteurs tels que Mwansoko (1990) et Samsom (1988). Ces auteurs montrent clairement dans leurs travaux comment les termes puristes développés dans la mécanique en Swahili ont été ignorés par le public qui leur a préféré les termes empruntés.

Nous pouvons aisément imaginer qu'il sera laborieux en termes de temps et de logistique pour mener à bien les enquêtes de terrain nécessaires. Ce qui est loin d'une affirmation gratuite de notre part comme on nous le fait souvent remarquer.

L'adoption de l'approche puriste n'est donc pas aisée dans un premier temps pour rendre disponible, dans des délais certains, une terminologie moderne des langues locales du Gabon. Dans un deuxième temps, cette approche ne réduira pas dans des délais acceptables l'écart de modernisation qui existe entre ces langues locales et le français afin que ces deux populations de langues soient enseignées de façon équitable.

C'est pour cela que nous, ainsi que de nombreux autres auteurs tels que Madiba (2001: 74), nous montrons relativement sceptiques par rapport à la création lexicale qui est un modèle plausible, mais qui implique un travail de recherche long et laborieux de termes en langue locales pour exprimer les con- 
naissances nouvelles et étrangères. Or, l'immense écart de terminologie moderne qu'il y a entre les langues locales africaines et européennes ne permet pas d'accuser encore des retards considérables. Comparaison est raison dans ce cas précis. En effet, il suffit de faire tout simplement comme cela l'a été dans toutes les langues du monde: emprunter à une ou plusieurs autres langues les mots manquants. Il s'agira ensuite de refléter cette mémoire et ce vécu des languesrécipients dans la confection des dictionnaires locaux en incorporant ces mots étrangers qui font dorénavant partie du patrimoine linguistico-culturel local comme entrées. Le dictionnaire est un ouvrage extensif, historisant, actuel et ouvert. Comme le dit Kalonji Zezeze (1993: 75) le dictionnaire est à la fois mémoire et vécu. Les populations locales du Gabon montrent la voie en empruntant au français les mots qui manquent dans leurs langues locales.

L'approche puriste, qui rappelons le semble être un processus contre nature dans le mécanisme de développement et de modernisation de la terminologie des langues, n'est pas un modèle adapté au développement et à la modernisation de la terminologie des langues locales du Gabon. Cette approche n'est donc pas également adaptée à la confection de dictionnaires locaux dans le traitement de la macrostructure dans lequel il faudra d'abord emprunter au français les mots qui manquent dans les langues locales.

L'approche qui doit être adoptée pour le développement et la terminologie des langues locales du Gabon ainsi que pour le traitement efficace de la macrostructure des dictionnaires locaux ne devrait pas être puriste ni même antipuriste également, mais «apuriste» pourrait-on dire. Autrement dit, une approche qui soit la plus neutre, mais aussi et surtout qui soit la plus objective possible en tenant compte des réalités du contexte dans lequel la langue est utilisée. Cette approche que nous avons déjà suggérée avant ces lignes se référera dans cette étude en termes d'approche pragmatique, cf. Madiba (2001).

\section{L'approche pragmatique dans le traitement de la macrostructure des dictionnaires gabonais}

\subsection{Approche définitoire}

Nous allons nous appuyer sur les travaux de certains auteurs afin de tenter de donner une définition de l'approche pragmatique dans le contexte précis du Gabon. Marshad (1984) et Mwansoko (1990) ont proposé les contours de la définition de l'approche pragmatique dans la modernisation du Swahili. Joseph (1984) a également indiqué quelques principes. À partir de ces orientations, nous définissons l'approche pragmatique dans le traitement de la macrostructure de dictionnaires locaux au Gabon, comme l'incorporation des mots empruntés au français dans le choix des mots à inclure comme lemmes ou entrées. Cette démarche n'est pas hasardeuse car, comme l'observe Ndong Ngoua (2007: 29), aucune langue n'est riche en elle-même. Bien au contraire, la langue est fonction du milieu physique, de l'organisation politique et sociale, 
du degré de technicité et d'emprunts aux autres langues. En effet, toutes les nations, soucieuses de domestiquer la science et ses applications, ont été confrontées aux mêmes difficultés que connaît le Gabon. Hier, c'était le cas des démocraties européennes telles que la France, l'Allemagne et la Grande Bretagne qui ont considérablement emprunté au latin et au grec. Aujourd'hui encore elles empruntent à l'anglo-américain qui devient la lingua franca. C'est aujourd'hui le cas des nouvelles puissances nucléaires, autrement dit l'Inde, le Pakistan et la Chine Populaire qui ont emprunté et adopté tous les mots internationaux scientifiques nécessaires même si elles sont d'origine occidentale, cf. Ndong Ngoua (2007: 31). Les pays émergents tels que le Gabon doivent également emboîter le pas de cette logique en s'accaparant de tous les mots internationaux scientifiques nécessaires qui sont dans le français pour enrichir et moderniser leurs langues locales.

\subsection{Les raisons de l'emprunt au français}

\subsubsection{Le français et les langues locales, langues partenaires et compagnons de route}

Le choix d'emprunter des mots issus du français dans le traitement de la macrostructure des dictionnaires locaux au Gabon n'est pas arbitraire. Comme le français et l'anglais, le français et nos langues locales sont de vieux compagnons de route 5 . Il y a même une relation d'emprunteurs mutuels entre ces langues comme c'est le cas entre le français et l'anglais. En effet, le français du Gabon qui est une variété du français standard en usage dans ce pays, quand bien même que celui-ci ne dispose pas encore d'une reconnaissance officielle, résulte de l'influence des langues locales du Gabon. L'approche qui consiste à emprunter des mots au français pour les intégrer dans la macrostructure des dictionnaires des langues locales du Gabon semble donc se présenter comme un choix d'emblée évident et naturel. En effet, le français et ces langues locales constituent deux populations de langues qui sont en contact depuis près d'un siècle. Même si ces deux populations de langues se distinguent par la famille $\mathrm{du}$ point de vue linguistique, il n'en demeure pas moins que le français fait maintenant partie entière du patrimoine linguistico-culturel du Gabon. Que certains linguistes africanistes gabonais le veuillent ou non, il faut nécessairement compter avec le français lorsqu'il est question du développement et de la modernisation des langues locales de ce pays. Le français est la langue de l'administration, de l'enseignement, de la mobilité sociale et du pouvoir du Gabon. Cette langue existe dans le quotidien et l'imaginaire des Gabonais car elle constitue la langue intermédiaire ou langue «hub». C'est-à-dire qu'au Gabon, le français est la langue véhiculaire qui permet la communication entre citoyens de groupes linguistiques différents. De par son omniprésence et son statut, il résulte aujourd'hui que le français est la langue maternelle d'un très grand nombre de Gabonais surtout au sein de la population jeune. Elle est de 
ce fait le substrat ${ }^{6}$ de nombreux Gabonais est la langue «compagnon» de leurs langues locales. Alors, ne pas tenir compte des mots du français qui sont utilisés, connus et familiers aux populations locales dans le traitement de la macrostructure des dictionnaires locaux au Gabon, ne serait pas une approche objective et naturelle dans le processus de développement et la terminologie des langues.

\subsubsection{L'emprunt au français comme la restitution d'un aspect de l'histoire du peuple gabonais dans les dictionnaires}

Un dictionnaire n'est pas seulement un ouvrage de référence contenant l'ensemble des mots d'une langue. Il inclut aussi des informations encyclopédiques essentielles qui apportent une connaissance sur notre histoire, une ouverture sur le monde et une réponse à toutes les curiosités des utilisateurs. Les dictionnaires traduisent, véhiculent et restituent tous les aspects de l'histoire des peuples. Les dictionnaires du Gabon doivent traduire et restituer l'étroitesse des relations qui existent entre le Gabon et la France. Ne pas considérer plusieurs mots du français comme lemmes ou entrées dans nos dictionnaires alors qu'ils sont abondamment utilisés, connus et familiers aux populations dans l'usage de leurs langues locales à l'oral, c'est nier une partie de l'histoire du Gabon. En effet, il s'agira donc de réfuter l'existence du français comme langue de la population en contact étroit avec les langues de ce pays. Cela déformerait peut-être pour ainsi dire assez considérablement une partie de l'histoire du Gabon dans laquelle notre pays est étroitement et intimement lié à la France. Nous avons souligné que comme le dit Kalonji Zezeze (1993: 75), le dictionnaire est à la fois mémoire et vécu. De ce point de vue, il nous paraît impossible de ne pas répertorier l'ensemble des faits linguistico-culturels de la société gabonaise dans laquelle le français est inclus et de s'en servir objectivement et naturellement pour développer et moderniser les langues locales de cette société.

\subsubsection{L'emprunt au français comme approche systématique du traitement de la macrostructure des dictionnaires gabonais}

La langue française est aujourd'hui une langue ouverte sur le monde car elle est faite d'influences mutuelles de diverses langues sur la scène politique, commerciale, économique, scientifique, technologique et sportive et plusieurs autres domaines encore aussi nombreux que divers. C'est donc une langue «mondiale» dont les termes que nous utilisons quotidiennement, auxquels les populations locales du Gabon sont accoutumées et qu'elles connaissent bien, nous aideront à moderniser assez facilement nos langues locales. Avoir cette langue dans le patrimoine linguistico-culturel du Gabon est une opportunité pour ce pays pour développer leurs langues et cultures locales. C'est de cette même façon 
que les démocraties européennes ont développé leurs langues en s'appuyant sur le latin et le grec dont les pays d'origine, à savoir Rome et la Grèce antique étaient des puissances prépondérantes.

Le français s'est aussi enrichi de nouveaux mots empruntés à l'Italie. Le 16ème siècle fut en effet marqué par la prépondérance de ce pays dans presque tous les domaines en raison de sa richesse économique, sa puissance militaire, son avance technologique et scientifique ainsi que sa suprématie culturelle. Cette influence, mais aussi la proximité ${ }^{7}$ entre la France et l'Italie se sont manifestées par la pénétration de quelque 8000 mots italiens dans la langue française. Il s'agissait notamment des termes relatifs à la guerre (canon, alarme, escalade, cartouche, etc.), à la finance (banqueroute, crédit, trafic, etc.), aux mœurs (courtisan, disgrâce, caresse, escapade, etc.), à la peinture (coloris, profil, miniature, etc.) et à l'architecture (belvédère, appartement, balcon, chapiteau, etc.), sans compter les domaines du vêtement, de l'alimentation, de l'équitation et de la musique.

Ignorer les mots du français qui ont pénétré le Gabon et que nous utilisons couramment afin de les inclure dans la macrostructure de nos dictionnaires pour obtenir ceux qui n'existent pas dans nos langues locales, serait à tout point de vue et sans trop grand risque de se tromper, adopter une approche non scientifique et objective. Ce sont plutôt des critères politiques, idéologiques et émotifs qui mettent en avant la sauvegarde de l'identité du Gabon ainsi qu'une volonté d'affirmer totalement l'indépendance vis à vis de la France.

Cependant, s'il est vrai que nous recommandons l'approche pragmatique qui consiste à emprunter au français les mots manquant aux langues locales afin de les inclure comme entrées dans la macrostructure, il est important de montrer comment les usagers vont intégrer ou adopter ces emprunts dans leurs habitudes linguistiques. C'est ainsi que dans les sections qui suivent, nous allons examiner en détail comment s'opèrent les deux phases de l'approche pragmatique dans le traitement de la macrostructure des dictionnaires gabonais. Tout au long de cet examen, nous mettrons en exergue les dispositifs spécifiques de la théorie et de la pratique de la lexicographie qui doivent caractériser les dictionnaires gabonais afin qu'ils soient de bonne qualité et qu'ils contribuent efficacement à l'effort de préservation et de promotion des nos langues locales. Il s'agit de la prévisibilité, de la calculabilité, de l'analysabilité et de la contrôlabilité.

\section{La phase de l'emprunt}

Cette phase se réfère à l'emprunt car elle consistera tout simplement à incorporer directement dans la macrostructure des dictionnaires de langues locales, les termes français qui n'ont pas officiellement d'équivalents dans ces langues. Il s'agira notamment aussi de conserver les termes modernes qui ont déjà des équivalents en langues locales.

Ces équivalents sont généralement de deux types de termes. Il y a d'une part les emprunts qui ont été substitués ou remplacés par des termes locaux et 
d'autre part, ceux qui ont subi des modifications pour se conformer aux structures linguistiques, c'est-à-dire, les structures morphologiques et phonologiques de ces langues. En fang ntoumou de Bitam comme dans presque toutes les langues locales du Gabon, ces deux catégories de mots apparaissent. Nous présentons ci-dessous quelques exemples en fang ntoumou de Bitam.

- Quelques mots substitués ou remplacés par des termes en fang ntoumou de Bitam

akôk (pile électrique), biyéne (lunettes), éloume (fourchette de table), mvet (guitare), nda mi mbok (prison), nda Zama (église), nkare fuègne (pasteur/ prêtre), nseng avion (aéroport), nseng ndama (stade/terrain de football), ofepeya (volant de voiture)

- Quelques mots ayant subi des modifications morphologiques et phonologiques

awola (heure, ou de l'anglais «hour»), dokira (médecin/titulaire d'un doctorat), kissine (cuisine ou de l'anglais «kitchen»), lama (lampe ou de l'anglais «lamp»), metoua (voiture, de l'anglais «motor»), nkolo (montre, de l'anglais «clock»), sikolo (école, études ou de l'anglais «school»), sobo (savon, de l'anglais «soap»), tawoula (serviette de bain, de l'anglais «towel»), tebele (table)

Ces mots que nous venons de prendre en exemple ci-dessus illustrent les types de termes locaux existant dans nos langues locales et qui représentent ou désignent des choses et des concepts ne provenant pas de nos sociétés locales. Ce sont à ces deux types de termes que seront donc ajoutés directement les termes empruntés au français qui seront aussi constitués de deux types. Il s'agit d'une part, de ceux que les populations locales utilisent quotidiennement dans leurs langues et qui leur sont par conséquent connus et familiers. Ce sont des mots tels que ciment, brique, goudron, banque, crédit, épargne, compte bancaire, béton, Député, Président, Ministre, Sénateur, fleur, Internet, informatique, géographie, cahier, crayon, carreau, règle, équerre, climatiseur, ordinateur, géographie, brique, salon, train, avion, hôtel, gaz, compas, taxi, livre, surfer (Internet), s'abonner, affecter (être), nommer (être), etc.

D'autre part, il s'agit essentiellement des mots du français qui appartiennent au vocabulaire des domaines spécialisés. Toutefois, les populations locales, étant donné leur familiarité avec le français, adoptent souvent facilement et rapidement ces termes dès qu'elles en prennent connaissance.

L'incorporation directe de ces mots empruntés au français, tout en conservant ceux qui en langues locales décrivent les concepts et choses modernes, permettra d'avoir des dictionnaires de nos langues locales dont les macrostructures vont présenter des vocabulaires modernes dans un temps relativement court avec un effet immédiat. En effet, les populations qui utilisent et connaissent déjà ces mots vont les adopter aussitôt. Par ailleurs, les mots qu'elles ne 
connaîtront pas, ne leur paraîtront pas totalement étrangers car ces populations connaissent déjà bien la langue française. En dehors de ces aspects, avec ces macrostructures, les lexicographes disposeront des listes de termes dont ils pourront dénombrer et analyser la quantité en fonction de ceux issus directement des emprunts, ceux issus des emprunts mais qui ont été phonologiquement et morphologiquement modifiés et ceux issus des langues locales. Cela conférera à ces dictionnaires gabonais les dispositifs spécifiques essentiels à la confection de bons dictionnaires selon les principes pratiques et théoriques de la métalexicographie que sont dans cette phase de l'emprunt, la calculabilité et l'analysabilité.

\section{La phase de substitution}

Dans le traitement de la macrostructure des dictionnaires de nos langues locales, la phase de substitution qui interviendra à court et à moyen termes consistera à remplacer progressivement tous les termes que les lexicographes ont empruntés au français sans savoir qu'ils existaient déjà dans la langue locale traitée. Cette procédure importante se réalisera grâce à l'interaction que les producteurs de dictionnaires doivent entretenir avec les locuteurs. Ils se mettront à la disposition des locuteurs pour recueillir ces mots, en s'assurant néanmoins que ceux-ci sont aussi effectivement reconnus par la communauté.

En effet, comme cela a été analysé par plusieurs lexicologues, l'expression populaire «maintenant, ce mot est dans le dictionnaire» est assez trompeuse. Elle semble bien montrer que les locuteurs, pendant un temps, ont l'intuition que tel mot n'est pas légitime, c'est-à-dire, qu'il «sonne» encore «étranger» et qu'il faut une autorité extérieure pour en déclarer le caractère français. En fait, le processus est inverse.

Les dictionnaires sont en réalité des instruments plus descriptifs que prescriptifs, qui ne font que sanctionner l'usage, quelle que soit la définition donnée à ce terme, et le représenter. Qu'un mot étranger entre dans le dictionnaire ne signifie pas qu'il ait été accepté seulement par une minorité compétente de linguistiques, grammairiens et lexicographes qui auraient le pouvoir de statuer sur la langue. C'est une image d'Epinal que la langue appartient aux locuteurs et aucun décret officiel ou théorie ne peut les contraindre à changer leurs usages du tout au tout. C'est ainsi qu'à travers les dictionnaires, les lexicographes ne font que signaler les usages qui sont devenus suffisamment courants. Cette première étape de la phase de substitution interviendra immédiatement dans la deuxième et la troisième édition revue et corrigée si nécessaire du dictionnaire confectionné. Aux dispositifs spécifiques lexicographiques de calculabilité et d'analysabilité de la phase de l'emprunt, va s'ajouter dans cette phase de l'emprunt, le dispositif spécifique de la contrôlabilité. L'informatique facilite aujourd'hui considérablement le travail du lexicographe. Avec les corpus électroniques, il sera facile de calculer, d'analyser et de contrôler le flux des termes locaux qui ont été sanctionnés par les locuteurs pour remplacer ceux qui 
ont été empruntés au français. Les dictionnaires de nos langues locales seront ainsi de bonne qualité à partir de la manipulation et de l'actualisation minutieuses de leurs textes. Cela permettra d'offrir régulièrement aux utilisateurs de nouvelles éditions dans des délais devenus de nos jours négligeables grâce à la facilité qu'offre aujourd'hui l'outil informatique dans le traitement de textes.

\subsection{La phase de l'actualisation de la création lexicale spontanée et natu- relle}

Dans l'approche pragmatique du traitement de la macrostructure des dictionnaires gabonais, la phase de l'actualisation de la lexicalisation locale va consister à intégrer régulièrement dans les éditions revues et corrigées, les termes empruntés qui ont été remplacés par les termes locaux. Comme nous l'avons mentionné plus haut, ces mots locaux ne sont pas en réalité nouveaux mais ce sont des extensions de sens qu'on leur a donnés et cela est tout à fait normal dans le fonctionnement de toute langue. Cette actualisation va se dérouler tout au long de l'histoire de notre pays. En nous référant aux propos d'Hagège (1987: 78), nous dirons l'évolution des parlers est aussi nécessaire aux lexiques que l'est à l'homme le renouvellement de l'air pur. Ainsi, au sein du lexique d'une langue, les glissements d'un sens à l'autre sont l'aventure permanente des mots pour accomplir la lourde et importante charge qu'ils ont, de refléter constamment les réalités complexes et changeantes de notre société.

Par l'action dynamique et naturelle des locuteurs dans leur intention de communiquer, ceux-ci attribuent des valeurs aux mots pour que les lexiques de leurs langues locales soient toujours adaptés à leurs besoins. Cela constitue d'ailleurs une des conditions du rayonnement d'une langue et se traduit par l'incorporation des vagues de mots migrants véhiculant de nouvelles choses et nouveaux concepts. Plusieurs de ces mots qui s'assimilent aux structures sémantiques, phonologiques et morphologiques de la langue récipiendaire seront digérés, les autres resteront tels qu'ils ont été empruntés. En fang ntoumou de Bitam par exemple, il s'agit dans le premier cas des termes tels que:

- akôk (pierre ou caillou) pour désigner «une pile électrique».

- amane ngouane (fin du mois) pour désigner «le salaire mensuel».

- me ayuèghène (des repos) pour désigner «les vacances».

- metoua wome a kouane/a bele okouane (ma voiture est malade/a une maladie), être malade ou avoir une maladie veut dire aussi être en panne ou avoir une panne en se référant à une automobile.

- metoua wome a ne wouane (ma voiture est en arrêt de panne), le mort désigne aussi un arrêt de panne de voiture.

- E Député wa a fe kousse biè e efefegne énguini a dza (Notre Député nous a acheté/offert un nouveau groupe électrogène au village). 
Dans le deuxième cas, il s'agit des mots que nous avons pris en exemple dans la section 4, cf. awola (heure/hour), ... tawoula (serviette de bain, de l'anglais «towel»), tebele (table).

Dans le troisième cas, ce sont les termes que nous avons aussi cités dans cette même section, cf. «ciment, brique, ... surfer (Internet), s'abonner, affecter (être), nommer (être), etc.

Nous allons nous intéresser précisément aux mots locaux qui ont été créés pour remplacer les mots empruntés. Les emprunts sont souvent spontanément et naturellement remplacés à long terme par les termes locaux lorsque les choses et les concepts étrangers exprimés par ces termes sont devenus communément connus au sein de la communauté (cf. Madiba 2001: 69). C'est le cas de certains mots remplacés par des termes en fang ntoumou de Bitam que nous avons présentés plus haut. C'est au vu de cette phase que Diop (1979: 345) rappelle à juste titre que le développement d'une langue est une entreprise de longue haleine qui est tantôt spontanée, tantôt confiée à des experts, sans que les deux modalités s'excluent.

En prenant akôk (pierre ou caillou), terme local fang ntoumou de Bitam pour désigner "pile électrique» comme exemple de mots locaux qui ont été créés pour remplacer les mots empruntés, un extrait de traitement bilingue français et fang ntoumou de Bitam se présentera ainsi comme suit:

$$
\text { pile [pil] } \mathbf{1} \mathrm{nf} \text { a (= tas) angosse } \mathrm{b} \text { [de pont] yini c (électrique) akôk... }
$$

En plus des dispositifs spécifiques de calculabilité, d'analysabilité et de contrôlabilité que vont acquérir les dictionnaires gabonais dont le traitement de la macrostructure va se réaliser en adoptant l'approche pragmatique plutôt que l'approche puriste, ces dictionnaires seront dotés du dispositif spécifique de prévisibilité. En effet, à partir des faits empiriques les lexicographes et linguistes pourront facilement déterminer les types de structures phonologiques et morphologiques des mots empruntés qui ont du mal à être intégrés dans nos langues, et leur prévoir ainsi une durée de vie limitée. C'est de la même façon que les spécialistes pourront assez aisément spéculer sur les mots empruntés qui seront remplacés par l'extension de sens d'un terme local ou ceux qui ne le seront jamais car ce sont des internationalismes 8 .

\subsection{Les raisons de la création lexicale spontanée et naturelle après l'emprunt}

Il est important de comprendre pourquoi la création lexicale intervient spontanément et naturellement pour remplacer plusieurs mots qui ont été empruntés. Cela est nécessaire pour que les personnes impliquées dans la confection de dictionnaires de nos langues, surtout celles qui sont favorables à l'approche puriste, comprennent le bien fondé de l'approche pragmatique qui régit de façon naturelle le fonctionnement des langues, et qu'il est fondamental de l'appliquer dans le traitement de la macrostructure de nos dictionnaires. Elles 
comprendront aussi et surtout que l'approche puriste que nous tous désirons en réalité car elle vise à donner une coloration et une identité locale à nos langues africaines, intervient spontanément et naturellement après avoir appliqué au préalable une approche pragmatique. Autrement dit, il faut en amont emprunter des mots internationaux scientifiques nécessaires inclus dans les langues occidentales.

\subsubsection{Le besoin d'une communication effective entre spécialistes, politiciens et analphabètes de la langue européenne}

Plusieurs raisons qui poussent spontanément et naturellement à la création lexicale pour remplacer les mots qui ont été aussi empruntés de la même manière ont été examinées à travers plusieurs travaux, notamment ceux de Marshad (1984), Sager (1989) et Mwansoko (1990).

Comme le souligne Madiba (2001: 70), les spécialistes ne rencontrent aucun problème dans l'usage des termes étrangers du moment où ces derniers possèdent de la matière en ce qui concerne la connaissance dans leur domaine de compétence et aussi une connaissance de la langue d'origine de ces mots. C'est aussi le cas pour les politiciens lors des campagnes électorales par exemple chères à notre pays, ainsi que des personnes en charge de la gestion de notre administration. Cependant, lorsqu'il est souvent question de transmettre cette connaissance en langue locale pour les besoins de communiquer avec un public qui ne maîtrise pas bien la langue européenne, le français dans le cas du Gabon, cela doit être fait en des termes locaux que ce public comprendra facilement. C'est dans ce type de circonstances ou de situations que les locuteurs et dans ce cas précis les spécialistes, administrateurs et politiciens locuteurs de leurs langues ou les personnes assignées à l'interprétation, créent spontanément et naturellement plusieurs mots qui permettront de bien transmettre le message et qui seront maintenant utilisés et acceptés par la communauté. C'est à partir de ce moment que ces mots locaux remplaceront les mots étrangers qui désignent les connaissances qui doivent être transmises.

Le besoin de développer des termes locaux résulte du fait qu'un spécialiste, un administrateur et un politicien ont souvent chacun une grande responsabilité envers sa communauté et doivent pour ce faire être capable de communiquer avec des analphabètes de la langue européenne, langue officielle. C'est face à cette exigence que de manière spontanée et naturelle le génie créateur des locuteurs permet d'élaborer des termes locaux qui remplaceront parfaitement les termes étrangers déjà empruntés ou non. Il ne restera plus alors au lexicographe qu'à les recueillir en vue du traitement de la macrostructure des dictionnaires de nos langues locales. C'est ainsi que dans le Département de la Mvoung par exemple, nous avons observé dans le vocabulaire politique l'usage de «édzikete» (ticket de confiance ou ticket qui donne le pouvoir) ${ }^{9}$ qui désigne le «bulletin de vote» et «ekete» (caisse) pour désigner l'«urne». Ces deux termes locaux font clairement comprendre l'importance et l'intérêt du vote aux popu- 
lations locales de cette localité. C'est dans ce même contexte que les personnes en charge de l'enrôlement ${ }^{10}$ qui se déroule actuellement dans toute l'étendue du territoire national au Gabon, ont initié un terme local, «e binenga e si» (s'enrouler par terre) car les populations locales connaissent plutôt «enrouler» ou «s'enrouler» et non «enrôler». Il ne serait pas donc étonnant que dans le vocabulaire politique d'Ovan, le terme «e binenga e si» deviennent la forme attestée pour signifier «s'enrôler».

Une création lexicale immédiate demandée aux spécialistes, administrateurs et politiciens ne donnera pas lieu au même résultat car il n'y aura pas le contexte favorable offert par l'interaction avec le public, l'acceptation et l'adoption de ce dernier des termes locaux utilisés à la place des termes étrangers.

\subsubsection{Durée de vie limitée de certains mots empruntés}

La deuxième raison pour laquelle la création lexicale remplace certains mots qui ont été empruntés, se situe dans la nature même de ces mots. Sager (1989) et Mwansoko (1990) relèvent que plusieurs mots empruntés ont une durée de vie limitée car ils sont dotés d'une nature telle qu'ils ont du mal à être assimilés au système linguistique de la langue récipiendaire. Après un certain temps d'usage, ce type de mots révèlent une existence précaire et sont par conséquent fréquemment remplacés. D'où, les limites de l'approche puriste à l'inverse de l'approche pragmatique, où il n'y aura pas le temps nécessaire pour que ce type de mots se révèlent d'eux-mêmes et soient spontanément et naturellement remplacés au fil du temps par les termes locaux.

\subsubsection{L'isolement des emprunts dans les énoncés}

La troisième raison qui conduit à la création lexicale après l'emprunt vient du fait que les mots étrangers intègrent souvent une langue de façon isolée et ne couvrent pas alors tout le concept qui gravite autour de lui (Madiba 2001: 7273). Cette nature individuelle fragilise l'existence des mots empruntés qui doivent en fait se fondre dans un énoncé pour donner des sens ou des idées plus complètes et plus générales qui vont au-delà des sens plus restreints véhiculés par ces mot. La phase de la création lexicale spontanée et naturelle se développe donc en essayant d'intégrer le mot emprunté dans un énoncé, c'està-dire, une structure conceptuelle plus élargie que ce mot seul. C'est ainsi que dans la phrase suivante en fang ntoumou de Bitam:

Be ve bere ma é tô. Be ve tuè me Directeur Général.

On m'a promu(e). On m'a placé(e)/nommé(e) Directeur Général.

En discutant avec un locuteur natif, il en ressort que le verbe "promouvoir» est complexe pour les populations locales. C'est ainsi que celui s'intégrait avec difficulté dans une phrase en fang ntoumou de Bitam dans laquelle il donne un 
sens à un énoncé dans cette langue. Dans ce cas précis, il s'agit d'une idée plus large ou plus complète dans le contexte d'une promotion professionnelle. Il a été mieux compris dans le sens de «élever», «augmenter» ou «faire monter», d'où «à bere» qui a ces sens dans ce dialecte fang du Gabon. L'intégration d'un emprunt dans un énoncé ne constitue donc pas un processus fragmentaire, les locuteurs essaient d'intégrer le concept véhiculé par ce mot dans un ensemble qui reflète les différentes structures énonciatives dans lequel ce mot joue un rôle déterminant dans le sens, cf. Sager (1989: 10).

C'est la raison pour laquelle les locuteurs remplacent spontanément et naturellement le mot étranger par un terme local une fois que le concept qu'il véhicule a été assimilé afin d'obtenir une communication plus effective avec des mots locaux qui sont plus clairs et évidemment accessibles. C'est ainsi que le verbe local «a bere» (faire monter, élever) a remplacé «promouvoir» pour que les fang ntoumou de Bitam comprennent facilement dans leur langue, comme c'est le cas, l'idée et l'acte contenus dans le verbe «promouvoir» dans une phrase ou un énoncé.

\subsubsection{Les limites des emprunts dans la production d'autres sens}

La dernière raison qui engendre souvent le besoin de recourir spontanément et naturellement à la création lexicale pour remplacer les mots empruntés, tient au fait que ces mots ne possèdent pas la même force productive que les termes locaux que les locuteurs maîtrisent mieux. Autrement dit, les locuteurs ne peuvent pas manipuler aussi bien les termes étrangers confinés à véhiculer un seul sens que leurs propres mots auxquels ils peuvent plus facilement ajouter d'autres nouveaux sens. Cette situation fragilise donc de nombreux mots qui sont empruntés et qui sont remplacés à terme par les mots locaux plus faciles à manipuler par les locuteurs.

\section{Les avantages de l'approche pragmatique dans le traitement de la macrostructure des dictionnaires gabonais}

\subsection{Approche systématique dans le traitement de la macrostructure des dictionnaires locaux au Gabon}

L'approche pragmatique que nous avons proposée dans cette étude présente plusieurs avantages. Le premier étant de reconnaître que l'emprunt et la création lexicale spontanée et naturelle de certains mots qui se déroulent à terme constituent deux aspects importants dans le traitement de la macrostructure des dictionnaires gabonais. Ce traitement dictionnairique aura également un impact positif sur le développement et la modernisation du vocabulaire des langues locales du Gabon, compte tenu du rôle et de l'importance du dictionnaire dans les langues. La phase de l'emprunt direct permet d'incorporer tous les mots manquants dans de délais de temps relativement courts et raison- 
nables, cf. Madiba (2001: 65).

Par ailleurs, l'emprunt va accroître les chances d'acceptabilité des termes empruntés au français. Ces termes que les populations rencontreront comme lemmes ou entrées des dictionnaires de langues locales leur seront déjà en réalité connus et familiers et seront utilisés. Comme le note Madiba (2001: 72), la plupart des emprunts pénètrent souvent une langue à travers les locuteurs eux-mêmes plutôt que par voie institutionnelle.

L'adoption de l'emprunt se révèle aussi importante pour rendre disponible auprès des populations des communautés ethnolinguistiques résidant dans les zones rurales de nouveaux concepts jusqu'ici inconnus à travers l'introduction des mots qui les représentent dans des dictionnaires qui leur sont destinés. La disponibilité de ces mots ainsi que de leurs significations conduira ces populations à se familiariser avec ces mots étrangers et les concepts qu'ils représentent. C'est à partir de cette familiarité qu'il sera possible à la communauté locale de créer des mots du terroir pour remplacer certains mots empruntés. D'où l'occasion de mentionner encore une fois les limites de l'approche puriste et les avantages de l'approche pragmatique. En effet comme l'indique Fodor (1975: 7), les termes locaux sont trouvés seulement après que les notions que chacun d'eux véhiculent soient sont devenues suffisamment familières au sein de la communauté et qu'il est aussi devenu clair que ces mots ne sont pas des effets de mode éphémère.

\subsection{La possibilité de la création lexicale spontanée et naturelle}

Le second avantage de l'approche pragmatique dans le traitement de la macrostructure des dictionnaires gabonais tient au fait qu'il offre la possibilité de réaliser après l'emprunt direct, une création lexicale spontanée et naturelle permettant de trouver des mots locaux qui seront acceptés et attestés par la communauté. C'est le cas des mots tels que akôk (pile électrique), biyéne (lunettes), éloume (fourchette de table), mvet(t) (guitare), nda mi mbok (prison), nda Zama (église), nkare fuègne (pasteur/prêtre), nseng avion (aéroport), nseng ndama (stade/terrain de football) et ofepeya (volant de voiture, de vélo et de moto) par exemple. Ces termes ont été créés spontanément par des locuteurs et de façon involontaire et inavouée, ces derniers termes sont acceptés par toute la communauté fang ntoumou de Bitam.

Ces quelques termes locaux que nous venons d'énumérer font rejoindre quelque peu l'approche puriste. À la différence que dans ce cas, cette approche puriste se déroule de façon plus conforme au processus naturel du développement et la modernisation des mots d'une langue. Dans ce «purisme», les locuteurs façonnent de façon naturelle leurs langues. Il nous plaît de signaler que nous souhaitons tous en réalité qu'une grande partie du vocabulaire de nos langues locales en Afrique soient celle du terroir. Cela est source de fierté, et aussi un marqueur et une manifestation importante de notre génie et de notre identité. Paradoxalement, ce résultat n'est possible que si au préalable les mots 
étrangers pénètrent ces langues afin que celles-ci les transforment en mots locaux.

\section{Conclusion}

Dans cet article, nous avons tenté de mettre en place une approche systématique dans le traitement de l'emprunt des mots au français dans la macrostructure des dictionnaires gabonais. Cette approche pragmatique s'est faite sur la base des dispositifs spécifiques des principes théoriques et pratiques de la lexicographie ainsi que sur la base des habitudes linguistiques des populations locales. C'est une image d'Épinal que la langue appartient aux locuteurs et la lexicographie tient compte de ce fait dans le traitement dictionnairique. C'est ainsi que nous avons essayé de montrer que l'approche pragmatique permettra de moderniser de façon systématique et élaborée le vocabulaire des langues locales à travers les dictionnaires et ce conformément aux habitudes des usagers pour qui ces ouvrages sont destinés. Cette approche permettra d'obtenir des ouvrages dans lesquels les populations se reconnaîtront et adopteront.

Les experts que nous sommes, linguistes, lexicographes et grammairiens, n'avons pas pour objectif de prescrire les langues locales en Afrique sans tenir compte des utilisateurs et des usages en cours de ces langues. Même si celles-ci incorporent des mots empruntés aux langues occidentales des anciennes puissances colonisatrices et que cela fait ressurgir les fantômes de la colonisation, il faudrait l'accepter ainsi. La présence de ces mots étrangers dans les langues locales d'Afrique est un processus inconscient et un facteur constitutif de la vie de toutes les langues du monde. Les langues-mères en Afrique n'échappent pas à ce fait et nous les experts ne devons pas faire de ces langues des exceptions. Il sera difficile à la minorité des experts que nous sommes de demander aux populations locales, par rupture immédiate ou progressive, de renoncer à l'utilisation des mots français pour satisfaire nos convictions idéologiques et politiques liées au désir de refouler un passé colonial douloureux. Ce passé a été certes douloureux mais nous ne pouvons pas refaire l'histoire. Nous devons vivre avec notre temps. Une marche en arrière n'est pas envisageable au nom de la recherche d'un pur pour d'identité.

Notre rôle n'est pas de produire des langues locales africaines selon nos idéologies et nos émotions. Nous devons d'une part les décrire telles qu'elles se manifestent et se pratiquent afin de mieux la comprendre et l'étudier. D'autre part, nous avons la mission d'organiser l'usage de la langue dans des ouvrages de base tels que les dictionnaires, les livres de grammaire et de lecture en conformité avec les besoins actuels afin de mieux les préserver et les promouvoir. Aucune langue ne naît ex nihilo par un pur pouvoir d'identité. Le développement d'une langue répond à une nécessité de construire et d'affermir l'État et la nation, de domestiquer la science et la technologie, de défendre et de faire rayonner la culture par des conceptions ou des pratiques qui apparaissent, cf. Ndong Ngoua (2007: 29). Par ailleurs, le traitement des langues locales dans les 
types d'ouvrages que nous avons cités plus haut permettra de les standardiser et les rendre conformes à l'enseignement.

Enfin, nous devons nous fonder sur les expériences accumulées sous d'autres cieux et du passé pour comprendre et apprendre comment le français par exemple s'est transformé d'un dialecte en une langue officielle et véhiculaire. Ces références nous permettront de mieux aborder le développement et la modernisation de nos langues africaines car elles ont bien plus de choses en commun que les langues occidentales, c'est-à-dire des universaux. Ne faisons pas de nos langues des objets scientifiques qui ne sont destinées qu'à la recherche fondamentale. Le fondement de toute langue est d'abord le prosaïsme qui résulte de la pratique linguistique tout aussi prosaïque des locuteurs qui la produisent depuis la nuit des temps sans critère scientifique. À force de présenter les langues africaines comme des objets scientifiques plutôt que des instruments pratiques qui servent à communiquer pour des raisons prosaïques, nous experts passons parfois à côté des besoins réels. Nombreux sont les locuteurs qui s'écartent de la scientificité qui est mise en avant leurs langues locales. Cette scientificité de ces langues que nous brandissons fait que les locuteurs pensent que leurs langues ne leur appartiennent plus, mais plutôt aux experts. Une des conséquences de ce caractère scientifique qui est octroyé aux langues africaines c'est que cela conduit les populations locales vers le français et l'anglais qui semblent plus accessibles et car n'étant pas perçus comme des objets scientifiques.

Nous espérons que cet article sera d'un apport considérable pour guider les personnes engagées dans la confection des dictionnaires de langues locales au Gabon ou dans d'autres pays africains francophones en particulier, afin que leurs projets aboutissent à la réalisation des dictionnaires de bonne qualité.

\section{Notes}

1. - Les États Généraux de l'Éducation et de la Formation, Libreville, mars 1983: «Les États généraux recommandent l'introduction de nos langues maternelles dans le système éducatif, et que les moyens en hommes et en matériels soient dégagés pour asseoir ou renforcer les structures de recherche appropriées à la réalisation progressive de cet objectif. Ils insistent pour qu'un conseil technique spécialisé se charge d'initier et de conduire les études en la matière en tenant compte des spécificités propres à notre pays.»

- Les États Généraux de l'Éducation: Projet de plan décennal 2010-2020 en matière d'Enseignement, de Formation et de Recherche. Libreville, 17-18 mai 2010: Introduction des langues locales comme activités d'immersion dans l'enseignement a été arrêtée comme une des actions et mesures inscrites dans le projet décennal issu de cette concertation.

2. Nous préviendrons ici qu'un reproche, une négation ou une critique acerbe au point de vue pratique et théorique, ne serait justifiable que si l'on occulte au Gabon l'origine historique, la place ainsi que le rôle des dictionnaires et de la lexicographie dans le développement et la modernisation des langues. Les dictionnaires sont confectionnés depuis près de 5000 av. J.-C. Par ailleurs, bien qu'ayant de domaine de recherche et de but différent, la confection de ces 
ouvrages était considérée comme une sous-discipline de la linguistique. Mais depuis la fin du 20ème siècle et le début du 21ème siècle, la lexicographie s'est établie comme une discipline autonome grâce aux travaux majeurs de Zgusta (1971), de Wiegand (1989a, 1989b, 1989c, 1992, 1996), de Tarp $(1998,2002)$, de Bergenholtz et Tarp (1995), de Bergenholtz, Tarp et Wiegand (1999), et de Landau (2001), pour ne citer que ces travaux-là parmi tant d'autres. Les caractéristiques essentielles du dictionnaire ainsi que les principes théoriques et pratiques sur lesquels reposent la confection du dictionnaire ne sont plus nécessaires à démontrer dans la préservation, la standardisation, la promotion et le développement des langues et cultures. C'est à ce titre que le dictionnaire est à la fois mémoire et vécu comme le souligne Kalonji Zezeze (1993: 75). Il était très important de signaler cet aspect dans ce travail. Au Gabon comme dans beaucoup de pays d'Afrique noire francophone, il existe une sorte de contexte préétabli et fort rigide où seul ne semble compter que l'approche de la linguistique africaniste. Les travaux des chercheurs lexicographes gabonais sont soumis à l'évaluation des linguistes africanistes gabonais ou étrangers. Dans ce contexte théorique assez particulier, le lexicographe africaniste en général, et gabonais en particulier semble (étrangement) jouer le rôle "usurpé" d'institution, cf. Kalonji Zezeze (1993: 85). Il lui est quasiment exigé ex abrupto de partir des travaux des chercheurs africanistes gabonais pour élaborer des manuels et des dictionnaires appropriés. Son approche est littéralement balayée d'un revers de la main comme si ses propositions étaient des hérésies. Cependant, l'approche ou les travaux des lexicographes gabonais reposent bel et bien sur les principes de la pratique millénaire et de la théorie actuelle de la lexicographie. La théorie et la pratique de la confection de dictionnaires ne peuvent être ramenées aux seuls aspects, recommandations, théories, méthodes et objectifs particuliers et parcellaires de la linguistique interne. Tout en reconnaissant le bien fondé de la linguistique africaniste, nous pensons que les problèmes linguistiques qui relèvent beaucoup plus de la recherche fondamentale, ne devraient pas occulter les aspects linguistiques pratiques et prosaïques qui sont fondamentaux dans la conception, l'élaboration et la fabrication de dictionnaires locaux au Gabon.

3. Nous signalons que nous n'avons pas présenté les transcriptions phonétiques car le traitement prosodique des langues locales du Gabon n'est pas l'objectif de ce travail. Par ailleurs, nous utilisons l'écriture disjonctive car nous avons observé que cette forme d'écriture sied mieux au traitement dictionnairique. En dehors de cela, elle sera plus accessible aux usagers lambdas et ceux du plus bas âge à cause des similarités avec le français, langue à laquelle les populations locales sont accoutumées. Le français est la langue officielle du Gabon. Elle est aussi langue de l'école, cf. Mba Nkoghé (2011: 7).

4. Nous sommes souvent assez surpris et surtout heurtés par la véhémence des observations de nombreux linguistes africanistes qui nous disent que "comparaison n'est pas raison" lorsque nous référons à l'histoire des autres langues pour mieux appréhender le développement des langues locales du Gabon. Quelle est cette hérésie scientifique de si grande ampleur commettons-nous dans une approche lexicographique, de vouloir tirer de l'expérience d'autres cieux des enseignements qui peuvent nous être utiles pour mieux appréhender une politique de préservation et de promotion de ces langues? "comparaison n'est pas raison" sont-ils des propos véritablement appropriés au domaine scientifique où "on ne réinvente pas la roue"? Nous ne pouvons pas traiter les langues locales du Gabon comme des cas isolés alors qu'elles ont subi et subissent encore les mêmes situations historiques, politiques, économiques et socioculturelles que les autres langues du monde. Comme dans le cas de l'anglais et du fran- 
çais par exemple, il s'agit au Gabon de deux populations de langues différentes, le français et les langues locales qui se côtoient à cause de leurs peuples qui sont en contact. Dans ce cas, il est inévitable qu'il y ait des échanges et des emprunts mutuels dans tous les aspects des activités de l'homme. Il devient nécessaire d'analyser les apports inévitables des uns et des autres. La langue en fait partie. Même si le français gabonais n'est ni scolarisé ni officialisé et ce malgré les appels de nombreux linguistes et lexicographes, il est bel et bien effectif. Il résulte de l'impact des langues locales sur le français standard. Le sens inverse est tout aussi effectif. Les langues locales du Gabon se sont enrichies de mots nouveaux en empruntant au français standard même si cela n'est pas officialisé. Quand bien même au point de vue linguistique le français et les langues locales du Gabon ne sont pas de la même famille, il est nécessaire d'étudier les échanges qu'il y a eu entre ces deux langues. C'est une obligation de voir comment cette interaction est utile pour la scolarisation et l'officialisation du français $\mathrm{du}$ Gabon et de celles des langues locales de ce pays. Ce ne sont pas des épiphénomènes comme cela est généralement présenté, de la recherche lexicologique de la linguistique africaine, pour servir d'esquisse à une lexicologie différentielle des langues locales du Gabon et du français. C'est ce dernier aspect qui fait partie de notre appel dans ce travail.

5. Plusieurs linguistes africanistes gabonais en particulier, ponctuent souvent d'"affirmation gratuite" nos propos lorsque nous évoquons ce sujet. Nous en sommes encore une fois surpris et profondément heurtés. Dans les pays d'Afrique noire, on vit un bilinguisme d'état généralisé dû en partie à l'instauration du nouvel ordre social issu du colonialisme, cf. Kalonji Zezeze (1993: 57). La question du français dans la vie nationale du Gabon donne lieu souvent à des débats que nous nous pouvons qualifier de stériles ou relevant de combats d'arrièregarde. Il est vrai que nous pouvons nous mettre aujourd'hui à penser ou à rêver de ce qu'aurait pu être nos langues locales si le français ne nous avait pas été imposé depuis un siècle. Malheureusement, nous ne pouvons pas refaire l'histoire. Nous devons vivre avec notre temps et assumer donc pleinement le français comme faisant partie de notre patrimoine linguistico-culturel. Nous affirmons et assumons entièrement ce fait ici car que ce statut soit officiel ou pas la théorie et la pratique lexicographique prend en compte le statut sociopolitique des langues en présence. De nombreux auteurs et personnes qui ne sauraient et pourtant assumer aujourd'hui l'avenir du Gabon si par rupture immédiate ce pays renonçait au français, se cachent derrière la réflexion universitaire et l'aveuglement politique ou idéologique et émotif pour avoir des réponses hypocrites à ce sujet. Cette hypocrisie s'observe aussi chez de nombreux linguistes africanistes d'origine européenne qui, perclus dans leurs rôle et mission avoués ou inavoués de "décolonisateurs", militent pour des langues locales d'Afrique qui soient pures, exemptes de tout élément de la langue de l'ancienne puissance colonisatrice. Nous les comprenons parfaitement car toute suggestion allant dans le sens d'utiliser les mots du français pour développer les langues locales leur donnerait à tort un rôle et une mission de "recolonisateurs".

6. Langue dont on repère les traces dans une autre langue et qui sert de base à cette dernière.

7. Après les guerres d'Italie qui s'étalèrent de 1494 à 1559, Français et Italiens tissèrent des liens étroits et pacifiques. De nombreux Italiens allèrent poursuivre leur carrière à la cour du roi de France et les mariages diplomatiques, comme celui de Catherine de Médicis avec Henri II, amenèrent à la cour des milliers d'intellectuels, d'artistes et de scientifiques italiens.

8. Les internationalismes peuvent être considérés comme les items lexicaux qui sont communs aux vocabulaires de plusieurs langues, cf. Braun (1989: 159). Toutefois, il faut également ajou- 
ter que pour qu'un item lexical soit considéré comme un internationalisme, il faudrait qu'il soit utilisé dans plusieurs «langues nationales, qu'il soit internationalement courant et qu'il soit compréhensible sans être traduit, cf. Braun (1989: 159). Sur cette base Braun (1989: 157) considère comme internationalismes, les items lexicaux suivants par exemple: Allemand (baseball, golf, cockpit, boxen, abstrakt, konkret, aktiv), Anglais (baseball, golf, cockpit, boxe, abstract, concret, active) et Français (baseball, golf, cockpit, boxe, abstrait, concret, actif).

9. Ces explications nous ont été fournies par Hidalgo Aboghe Nzome, journaliste en poste à RTG 6 à Makokou, Province de l'Ogooué Ivindo.

10. Il s'agit de Mekeng Edouard, Enseignant du secondaire (Histoire et Géographie), et d'Endama Urcy, Technicien à Gabon-Poste. Ils sont respectivement Représentant de la Majorité et Opérateur dans la Commission d'Enrôlement pour l'Identité Biométrique du Gabon (IBOGA) pour le compte de la Commune d'Ovan.

\section{Bibliographie}

Bergenholtz, H. et S. Tarp (Éds.). 1995. Manual of Specialised Lexicography: The Preparation of Specialised Dictionaries. Amsterdam/Philadelphia: John Benjamins.

Bergenholtz, H., S. Tarp et H.E. Wiegand. 1999. Datendistributionsstrukturen, Makro- und Mikrostrukturen in neueren Fachwörterbüchern. Fachsprachen. Ein internationales Handbuch zur Fachsprachenforschung und Terminologiewissenschaft/Languages for Special Purposes. An International Handbook of Special-Language and Terminology Research. Volume 2: 1762-1832. Berlin/New York: Walter de Gruyter.

Braun, P. 1989. Internationalisms: Identical Vocabularies in European Languages. Coulmas, F. (Éd.). Language Adaptation: 158-167. Cambridge: Cambridge University Press.

Diop, C.A. 1979. Nations nègres et culture. Tome 2. Paris: Présence Africaine.

Fodor, I. 1975. Language Reforms of the Past and in the Developing Countries. Herbert, R.K. (Éd.). 1975. Patterns in Language, Culture and Society: Sub-Saharan Africa: 3-17. Columbus: The Ohio State University.

Gouws, R.H. 2001. Formation lexicographique: approches et thèmes. Emejulu, J.D. (Éd.). 2001. Éléments de lexicographie gabonaise. Tome I: 95-134. New York: Jimacs-Hillman.

Hagège, C. 1987. Le français et les siècles. Paris: Odile Jacob.

Hausmann, F.J et al. (Éds.). 1989-1991. Wörterbücher. Ein internationales Handbuch zur Lexikographie/ Dictionaries. An International Encyclopedia of Lexicography / Dictionnaires. Encyclopédie internationale de lexicographie. Berlin: Walter de Gruyter.

Jernudd, B.H. 1977. Linguistic Sources for Terminological Innovation: Policy and Opinion. Rubin, J., B.H. Jernudd, J. Das Gupta, J.A. Fishman et C.A. Ferguson (Éds.). 1977. Language Planning Processes: 215-236. Le Havre: Mouton.

Joseph, J. 1984. The Elaboration of an Emerging Standard. Language Sciences 6(1): 39-52.

Kalonji Zezeze, M.T. 1993. La lexicographie bilingue en Afrique francophone: L'exemple français-cilubà. Paris: L'Harmattan.

Landau, S.I. 2001. Dictionaries: The Art and Craft of Lexicography. New York/Cambridge: Cambridge University Press.

Madiba, M.R. 2001. Towards a Model for Terminology Modernisation in the African Languages of South Africa. Language Matters 32: 53-77. 
Marshad, H.A. 1984. An Approach to Code Elaboration and its Application to Swahili. Thèse de doctorat non publiée. Urbana-Champaign: University of Illinois.

Mba Nkoghé. 2011. La langue de l'école. Gabon Matin, Quotidien d'informations générales No. 590: 7.

Mwansoko, H.J.M. 1990. Modernisation of Swahili Technical Terminologies: An Investigation of the Linguistics and Literature Terminologies. Thèse de doctorat non publiée. York: University of York.

Ndong Ngoua, A. 2007. L'officialisation des langues africaines: Une question de volonté politique. Revue semestrielle de l'Institut de Recherche en Sciences Humaines (CENAREST-Gabon) 11-12: 21-30.

Sager, J.C. 1989. Terminological Problems Involved in the Process of Exchange of New Technology between Developing and Developed Countries: Study on Recent Developments in the Relationship between Science, Technology and Society in Different Economic, Social and Cultural Contexts. Paris: UNESCO.

SALIC (Service de L'Aménagement Linguistique du Canada). Histoire de la langue française. http: //www.salic.slmc.ca/.

Samsom, R.H. 1988. Ufundi (wa) magari: mfano wa ukuzaji wa istalahi za Kiswahili nchini Tanzania matumizi wa usanifishaji wake. M.A. thèse non publiée. Leyde: Université de Leyde.

Smit, M. 1996. Wiegand's Metalexicography as a Framework for a Multilingual, Multicultural, Explanatory Music Education Dictionary for South Africa. Thèse de doctorat non publiée. Stellenbosch: Université de Stellenbosch.

Tarp, S. 1998. Leksikografien på egne ben. Fordelingsstrukturer og byggedele i et brugerorienteret perspektiv. Hermes, Journal of Linguistcs 21: 121-137.

Tarp, S. 2002. Translation Dictionaries and Bilingual Dictionaries - Two Different Concepts. Journal of Translation Studies 7: 59-84.

Weinstein, B. 1989. Francophonie: Purism at the International Level. Jernudd, B.H. et M.J. Shapiro (Éds.). 1989. The Politics of Language Purism: 53-79. Berlin: Mouton de Gruyter.

Wiegand, H.E. 1989a. Arten von Mikrostrukturen im allgemeinen einsprachigen Wörterbuch. Hausmann, F.J. et al. (Éds.). 1989-1991: 462-501.

Wiegand, H.E. 1989b. Aspekte der Makrostruktur im allgemeinen einsprachigen Wörterbuch: Alphabetische Anordnungsformen und ihre Probleme. Hausmann, F.J. et al. (Éds.). 1989-1991: 371-409.

Wiegand, H.E. 1989c. Der gegenwärtige Status der Lexikographie und ihr Verhältnis zu anderen Disziplinen. Hausmann, F.J et al. (Éds.). 1989-1991: 246-280.

Wiegand, H.E. 1992. Elements of a Theory towards a So-called Lexicographic Definition. Lexicographica 8: 175-289.

Wiegand, H.E. 1996. A Theory of Lexicographic Texts. An Overview. South African Journal of Linguistics 14(4): 134-149.

Zgusta, L. 1971. Manual of Lexicography. Le Havre: Mouton. 